\title{
Numerical Study of the Effect of Magnetic Field on Nanofluid Heat Transfer in Metal Foam Environment
}

\author{
Hamid Shafiee $\left(\mathbb{D},{ }^{1}\right.$ Elaheh NikzadehAbbasi, ${ }^{2}$ and Majid Soltani ${ }^{3,4,5,6}$ \\ ${ }^{1}$ Department of Mechanical Engineering, Shahid Bahonar University of Kerman, Kerman, Iran \\ ${ }^{2}$ Department of Chemical Engineering, Shahid Bahonar University of Kerman, Kerman, Iran \\ ${ }^{3}$ Department of Mechanical Engineering, K.N. Toosi University of Technology, Tehran, Iran \\ ${ }^{4}$ School of Optometry and Vision Science, Faculty of Science, Department of Electrical and Computer Engineering, \\ Faculty of Engineering, University of Waterloo, Waterloo, Canada \\ ${ }^{5}$ Waterloo Institute for Sustainable Energy (WISE), University of Waterloo, Waterloo, Ontario, Canada \\ ${ }^{6}$ Advanced Energy Initiative Center, K.N. Toosi University of Technology, Tehran, Iran \\ Correspondence should be addressed to Hamid Shafiee; h.shafiee@eng.uk.ac.ir
}

Received 28 August 2021; Revised 2 October 2021; Accepted 18 October 2021; Published 8 November 2021

Academic Editor: Jianchao Cai

Copyright (C) 2021 Hamid Shafiee et al. This is an open access article distributed under the Creative Commons Attribution License, which permits unrestricted use, distribution, and reproduction in any medium, provided the original work is properly cited.

\begin{abstract}
The magnetic field can act as a suitable control parameter for heat transfer and fluid flow. It can also be used to maximize thermodynamic efficiency in a variety of fields. Nanofluids and porous media are common methods to increase heat transfer. In addition to improving heat transfer, porous media can increase pressure drop. This research is a computational simulation of the impacts of a magnetic field induced into a cylinder in a porous medium for a volume fraction of 0.2 water/ $\mathrm{Al}_{2} \mathrm{O}_{3}$ nanofluid with a diameter of $10 \mu \mathrm{m}$ inside the cylinder. For a wide variety of controlling parameters, simulations have been made. The fluid flow in the porous medium is explained using the Darcy-Brinkman-Forchheimer equation, and the nanofluid flow is represented utilizing a two-phase mixed approach as a two-phase flow. In addition, simulations were run in a slow flow state using the finite volume method. The mean Nusselt number and performance evaluation criteria (PEC) were studied for different Darcy and Hartmann numbers. The results show that the amount of heat transfer coefficient increases with increasing the number of Hartmann and Darcy. In addition, the composition of the nanofluid in the base fluid enhanced the PEC in all instances. Furthermore, the PEC has gained its highest value at the conditions relating to the permeable porous medium.
\end{abstract}

\section{Introduction}

The most important parameters discussed in heating systems and industrial centers are the increase of heat transfer and advanced and optimal cooling [1]. Optimization of heat transfer systems to increase the heat flux in most thermal equipment is possible by increasing the surface and then increasing the volume and size of the equipment [1]. Helps to cool the tool and workpiece (cooling property), reduces wear and friction between the tool and the workpiece (lubrication property), transfers chips outside the cutting area (liquid flow property), prevents heat transfer from the tool and workpiece to Gates (heat absorption property), and separates cutting debris from the part and tools (increasing surface quality) are the advantages of shear fluid [2]. Nano- fluid technology has attracted the attention of many researchers due to its thermal conductivity and higher heat transfer ability than conventional heat transfer fluids or fluids containing particles in microdimensions [3]. By using nanofluids in industrial equipment, thermal performance can be significantly increased, in which case a large benefit will be given to industrial units both in terms of energy savings and in terms of better equipment performance [4, 5]. Liquids are commonly used in industry for heat transfer and cooling. The growth and development of industry and manufacturing activities have led researchers and manufacturers to take a fresh look at heat transfer and cooling fluid methods [5]. Nanofluids are a new kind of fluids derived from the distribution of nanosized particles in regular fluids. They have many possibilities for industrial uses. Nanofluids 
employ particles of sizes ranging from $1 \mathrm{~nm}$ to $100 \mathrm{~nm}$. Metal particles like copper $(\mathrm{Cu})$, silver (silver), and metal oxides such as aluminum oxide $\left(\mathrm{Al}_{2} \mathrm{O}_{3}\right)$ and copper oxide $(\mathrm{CuO})$ make up these particles $[6,7]$. The thermal conductivity of conventional heat transfer fluids is low. The thermal conductivity of the fluid is improved by distributing nanoparticles in the base fluid, which is one of the key elements in heat transfer, resulting in high conductivity [8].

Heat transfer has always been one of the significant challenges in engineering. Many methods have been proposed to increase or control heat transfer. One of these methods is the use of nanofluids for heat transfer [9]. On the other hand, recognizing nanofluid flow in the presence of external fields and the impact of external fields on the nanofluid heat transfer rate is the subject of much discussion in engineering and medical sciences. Magnetic fields are one of the external fields applied to the fluid, which has been considered in recent decades due to its special properties. Many works have been performed on the impact of such fields on flow characteristics, heat transfer, and other fluid transfer properties. Most research has a theoretical and numerical basis, and considerable experimental work has not been done in alternating magnetism and heat transfer, especially in the field of slow current, which has its application [10]. Cooling devices are among the essential concerns of factories and industries and everywhere that somehow face heat transfer. The usage of modern and optimal cooling technologies is unavoidable in these circumstances. The most common technique to improve current heat transfer devices is to expand their surface area, which always raises their volume and size. Therefore, new and effective coolers are needed to overcome this problem. Nanofluids have been proposed as a new solution in this field. In terms of the effect of a magnetic field on slow flow nanofluid heat transfer, the problem can be studied from two perspectives. First, the magnetic effect is added to the Navier-Stokes equation, which shows the magnetic effect as an external force. The second view is the effect that the magnetic field has on the physical properties of the nanofluid [11].

In recent years, according to laboratory studies on the effect of the magnetic field on the viscosity and specific heat capacity of biphasic fluids such as nanoparticles, the results show that the observed changes may be due to the presence of particles in the fluid or the change in fluid flow behavior applied in the presence of a magnetic field. Also, laboratory and computational studies on the effect of magnetic waves as a targeted drug transfer agent in the body attempted to study various factors affecting motion using experimental and numerical work and heat transfer of nanofluids in the presence of magnetic fields. In this numerical research, new methods to increase the heat transfer coefficient, such as magnetic field application and injection of nanoparticles, are simulated, and their efficiency is determined.

Dukhan and Chen [12] conducted a study examining the heat transfer inside a sample of the commercially available metal foam heat sink, which is given a constant heat flux by an electronic device. They found from the empirical findings that as the distance from the heated surface rises, its temperature decreases rapidly. Calmidi and Mahajan [13] obtained the effective conduction coefficient of conductivity for aluminum foam in one study. The effective heat transfer coefficient is a property of metal foam that measures the ability of a metal foam to transfer heat through the air and the solid metal part in the state of free flow. In recent years, many research groups have tried to study and apply these porous materials from a practical point of view. Boyd and Hooman [14] designed and studied a model for studying the power of using metal foams in interconnected fuel cells. They found from this study that a heat exchanger made of metal foam with an air-cooled fluid with the same pressure difference could be an excellent alternative to the same sample with a water-cooled fluid. Odabaee and Hooman [15] conducted a study to replace heat exchangers with metal foams instead of finned tubes in water-cooled condensers. In this study, they concluded that using these metal foams can have 2 to 6 times better performance in terms of heat transfer (to increase the pressure drop is reasonable and acceptable). Lin et al. [16] also studied heat exchangers. They studied six samples of heat exchangers, 3 of which were made of copper metal foam and the other three samples of nonporous heat exchangers made of copper. In this experiment, they found that copper foam has a higher heat transfer and a lower pressure drop. Several strategies for increasing heat transfer from an item have been presented. Using a fluid with better heat transfer characteristics is one of these techniques. Nanofluids are a new category of fluids with superior thermal properties over conventional fluids. These fluids are made up of a base fluid and nanometer-sized particles. These particles, usually metals, metal oxides, or nanotubes, boost the coolant's conductivity and heat transfer coefficient.

Chen et al. [17] studied the natural convective heat transfer investigation of nanofluids affected by the electrical field. The heat transfer performance of resuspended nanofluid under the electric field is significantly increased compared to the pure base fluid, which increases with the improvement of voltage, concentration, and direction change time. Qi et al. [18] investigated the effects of rotation angle and metal foam on the natural convection of nanofluids in a cavity under an adjustable magnetic field. They showed that the horizontal magnetic field is not essential for increased thermal performance. However, the vertical magnetic field shows an opposite trend and has a positive contribution to thermal performance. The cavity with a rotation angle $\alpha=90$ degrees shows the highest thermal performance. Izadi et al. [19] study on impingement cooling of a porous metal CPU cooler saturated with nanofluid under the magnetic field effects. The results show that increasing the Darcy number can increase the heat transfer performance. At the same time, contrasting trends are found for the aspect ratio and the Eckert number. The Rabbani et al. [20] study experimentally investigated the thermal performance and the pressure drop in copper tubes partially filled with open-cell metal foams using $\mathrm{MgO}$ nanofluid. It has been observed that the pressure drop and the Nusselt number depend significantly on the Reynolds number. Further results show that nanofluid and porous media inside the 
tubes significantly increase the pressure drop and the heat transfer coefficient. Ameri et al. [21] studied the use of a single-phase method modified with a thermal dispersion model that includes a heterogeneous distribution of nanoparticle concentrations to evaluate the thermal performance in a porous foam tube. The results show that the concentration heterogeneity is proportional to the metal foam's mean nanoparticle concentration, Reynolds number, and porosity. The velocity and temperature cross-sectional profiles in the dispersion model are flatter than the samples obtained from the homogeneous model. In addition, it is found that the Nusselt number differs directly from the mean concentration and the Reynolds number. At the same time, the inverse ratio for porosity varies. This reduction is in less deep porosities. Badfar et al. [22] studied the use of drug-coated ferrite nanoparticles to target magnetic drugs to the stenosis area of the conduit. For varied magnetic numbers, the issue was solved. The impact of the wire's position as a magnetic field on the MDT also was investigated. Previous studies have investigated the increase in heat transfer in porous media when nanofluids are used. The magnetic field is activated separately and in combination. Studies have shown that a few previous studies have investigated the forced displacement heat transfer and the nanofluid magnetic field within a cylinder in a porous medium, taking into account Darcy and Hartmann numbers. In this research, simultaneously, the impact of the magnetic field's presence, nanoparticles, and porous medium on heat transfer will be investigated numerically. Aluminum oxide $\left(\mathrm{Al}_{2} \mathrm{O}_{3}\right)$ nanoparticles and metal foam nickel $(\mathrm{Ni})$ are considered. The effect of nanoparticle concentration and the intensity and direction of magnetic field application will be investigated. The results will be analyzed using Nusselt number, pressure drop, average speed, velocity, and outlet temperature.

\section{Methods and Material}

2.1. Problem Geometry. Figure 1 schematically shows the forced heat transfer for $\mathrm{Al}_{2} \mathrm{O}_{3}$ nanofluid inside a twodimensional cylinder (symmetrical axis view) with a magnetic field angle of $0^{\circ}<\Psi<90^{\circ}$. This schematic includes a pipe with a length of $0.3 \mathrm{~m}$ and a diameter of $0.01 \mathrm{~m}$. Pure water or $\mathrm{Al}_{2} \mathrm{O}_{3}$ nanofluid, which enters the tube in a gentle axial direction with the same entrance velocity and $U_{\text {in }}$ and $T_{\text {in }}$ temperatures, is the fluid inside the tube. This paper used water and aluminum nanofluids $\left(\right.$ water $/ \mathrm{Al}_{2} \mathrm{O}_{3}$ ) with a volume fraction of 0.2 nanofluids. Also, the diameter of aluminum nanofluid is equal to 10 micrometers. A uniform magnetic field of magnitude B0 was also applied to the fluid flow. This research is aimed at comparing the thermal efficiency of the nanofluid in question to that of the base fluid. The impact of variables like Darcy and Hartmann numbers was also investigated. The problem is simulated in several different modes to investigate the magnetic field, the porous medium, and the impact of the nanofluid (water and $\mathrm{Al}_{2} \mathrm{O}_{3}$ nanofluid). Different Darcy numbers $(0.1,0.01,0.001$, and 0.0001$)$ have been investigated to investigate the porous medium.
Also, different Hartmann numbers (10, 20, 30, and 40) and different magnetic field orientations $(0,30,60$, and 90 degrees) have been investigated to investigate the magnetic field. Nickel is also thought to be present in a porous metal foam environment.

2.2. The Governing Equations. The equations of mass, momentum, and energy for a $2 \mathrm{D}$ steady-state flow are shown here:

$$
\begin{aligned}
\frac{\partial u}{\partial x}+\frac{\partial v}{\partial y}= & 0 \\
\frac{1}{\mathcal{\varepsilon}^{2}}\left(u \frac{\partial u}{\partial x}+v \frac{\partial u}{\partial y}\right)= & -\frac{1}{\rho_{n f}} \frac{\partial p}{\partial x}+\frac{\vartheta_{n f}}{\varepsilon}\left(\frac{\partial^{2} u}{\partial x^{2}}+\frac{\partial^{2} u}{\partial y^{2}}\right) \\
& +\frac{\sigma_{n f} B_{0}^{2}}{\rho_{n f}}\left(v \sin (\psi) \cos (\psi)-u \sin ^{2}(\psi)\right) \\
& -\frac{\vartheta_{n f} u}{K}-\frac{\vartheta_{n f} C_{d}|\vec{u}|}{\sqrt{k}} u \\
\frac{1}{\varepsilon^{2}}\left(u \frac{\partial v}{\partial x}+v \frac{\partial v}{\partial y}\right)= & -\frac{1}{\rho_{n f}} \frac{\partial p}{\partial y}+\frac{\vartheta_{n f}}{\varepsilon}\left(\frac{\partial^{2} v}{\partial x^{2}}+\frac{\partial^{2} v}{\partial y^{2}}\right) \\
& +\frac{\sigma_{n f} B_{0}^{2}}{\rho_{n f}}\left(v \sin (\psi) \cos (\psi)-v \cos ^{2}(\psi)\right) \\
& -\frac{\vartheta_{n f} v}{K}-\frac{\vartheta_{n f} C_{d}|\vec{v}|}{\sqrt{k}} v,
\end{aligned}
$$

$$
u \frac{\partial T}{\partial x}+v \frac{\partial T}{\partial y}=\alpha_{n f}\left(\frac{\partial^{2} T}{\partial x^{2}}+\frac{\partial^{2} T}{\partial y^{2}}\right)
$$

Several variables in dimensionless equations are described as follows:

$$
\begin{aligned}
X & =\frac{x}{D}, \\
Y & =\frac{y}{D}, \\
U & =\frac{u}{U_{\text {in }}}, \\
V & =\frac{v}{U_{\text {in }}}, \\
P & =\frac{p}{\rho U_{\text {in }}^{2}}, \\
\theta & =k_{f} \frac{T-T_{\text {in }}}{q^{\prime \prime} D},
\end{aligned}
$$




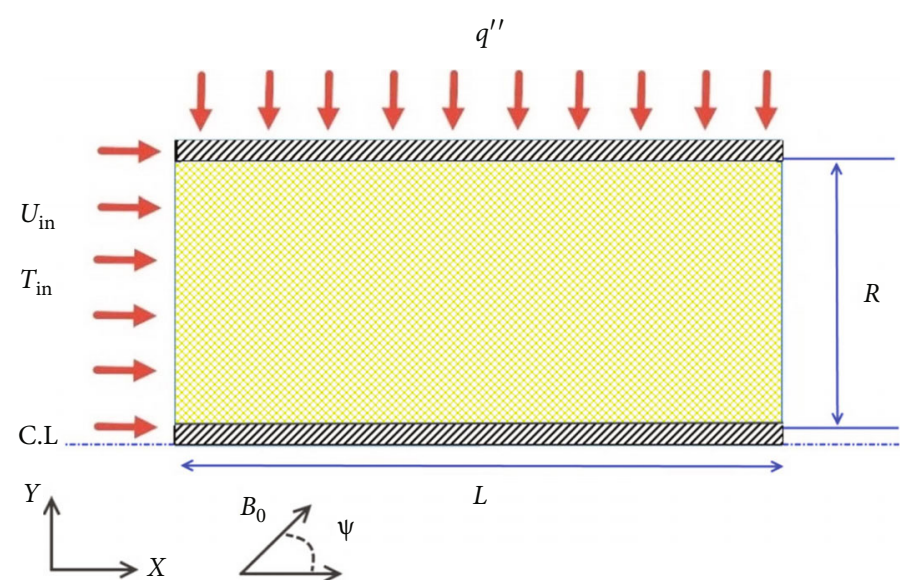

Figure 1: Schematic showing the problem that was resolved.

$$
\begin{aligned}
& C_{d}=\frac{1.75}{\sqrt{150} \varepsilon^{3 / 2}}, \\
& \operatorname{Pr}=\frac{\vartheta_{f}}{\alpha_{f}}, \\
& \operatorname{Re}=\frac{\rho_{f} U D}{\mu_{f}}, \\
& \mathrm{Nu}=\frac{h D}{k}, \\
& \mathrm{Da}=\frac{k}{D^{2}}, \\
& \mathrm{Ha}=B_{o} D \sqrt{\frac{\sigma_{f}}{\mu_{f}} .}
\end{aligned}
$$

In Equation (3), $C_{d}, \mathrm{Pr}, \mathrm{Re}, \mathrm{Nu}, \mathrm{Da}$, and $\mathrm{Ha}$ denote the inertial coefficients of the porous medium, Prantel, Reynolds, Nusselt, Darcy, and Hartmann, respectively. The dimensionless form of the equations may be derived by replacing the dimensionless parameters in

$$
\begin{gathered}
\frac{\partial U}{\partial X}+\frac{\partial V}{\partial Y}=0 \\
\frac{1}{\varepsilon^{2}}\left(U \frac{\partial U}{\partial X}+V \frac{\partial U}{\partial Y}\right)=-\frac{\rho_{f}}{\rho_{n f}} \frac{\partial P}{\partial X}+\frac{\vartheta_{f}}{\vartheta_{n f}} \frac{1}{\operatorname{Re} \cdot \varepsilon}\left(\frac{\partial^{2} U}{\partial X^{2}}+\frac{\partial^{2} U}{\partial Y^{2}}\right) \\
+\frac{\rho_{f}}{\rho_{n f}} \frac{\sigma_{n f}}{\sigma_{f}} \frac{\mathrm{Ha}^{2}}{\operatorname{Re}}\left(V \sin (\psi) \cos (\psi)-U \sin ^{2}(\psi)\right) \\
-\frac{U}{\operatorname{ReDa}}-\frac{\varepsilon C_{d}|\vec{U}|}{\sqrt{\mathrm{Da}}} U
\end{gathered}
$$

$$
\begin{aligned}
\frac{1}{\varepsilon^{2}}\left(U \frac{\partial V}{\partial X}+V \frac{\partial V}{\partial Y}\right)= & -\frac{\rho_{f}}{\rho_{n f}} \frac{\partial P}{\partial Y}+\frac{\vartheta_{f}}{\vartheta_{n f}} \frac{1}{\operatorname{Re} \cdot \varepsilon}\left(\frac{\partial^{2} U}{\partial X^{2}}+\frac{\partial^{2} U}{\partial Y^{2}}\right) \\
& +\frac{\rho_{f}}{\rho_{n f}} \frac{\sigma_{n f}}{\sigma_{f}} \frac{\mathrm{Ha}^{2}}{\operatorname{Re}}\left(U \sin (\psi) \cos (\psi)-V \sin ^{2}(\psi)\right) \\
& -\frac{V}{\operatorname{ReDa}}-\frac{\varepsilon C_{d}|\vec{V}|}{\sqrt{\mathrm{Da}}} V
\end{aligned}
$$

$$
U \frac{\partial T}{\partial X}+V \frac{\partial T}{\partial Y}=-\frac{k_{n f}}{k_{f}} \frac{1}{\operatorname{Re} \cdot \operatorname{Pr}} \frac{\left(\rho c_{p}\right)_{f}}{\left(\rho c_{p}\right)_{n f}}\left(\frac{\partial^{2} \theta}{\partial X^{2}}+\frac{\partial^{2} \theta}{\partial Y^{2}}\right)
$$

In recent decades, the physical properties of nanofluids have received more attention. In this study, water was considered the main fluid in which aluminum particles were used as the most common nanoparticles. The following equations have been used to determine the effective coefficients of electrical conductivity, density, volumetric thermal expansion, heat capacity, and thermal penetration of the studied nanofluid:

$$
\begin{aligned}
\sigma_{\mathrm{hnf}} & =(1-\phi) \sigma_{\mathrm{f}}+\phi \sigma_{\mathrm{np}}, \\
\rho_{\mathrm{hnf}} & =(1-\phi) \rho_{\mathrm{f}}+\phi \rho_{\mathrm{np}}, \\
(\rho \beta)_{\mathrm{hnf}} & =(1-\phi)(\rho \beta)_{\mathrm{f}}+\phi(\rho \beta)_{\mathrm{np}}, \\
\left(\rho c_{P}\right)_{\mathrm{hnf}} & =(1-\phi)\left(\rho c_{P}\right)_{\mathrm{f}}+\phi\left(\rho c_{P}\right)_{\mathrm{np}}, \\
\alpha_{\mathrm{hnf}} & =\frac{k_{\mathrm{hnf}}}{\left(\rho c_{P}\right)_{\mathrm{hnf}}} .
\end{aligned}
$$

In Equations (8)-(12), hnf, $\mathrm{f}$, and $\mathrm{np}$ represent the properties of composite nanofluids, pure fluids, and nanoparticles, respectively. Maxwell and Brinkmann equations were used to calculate the thermal conductivity and viscosity of the nanofluid: 


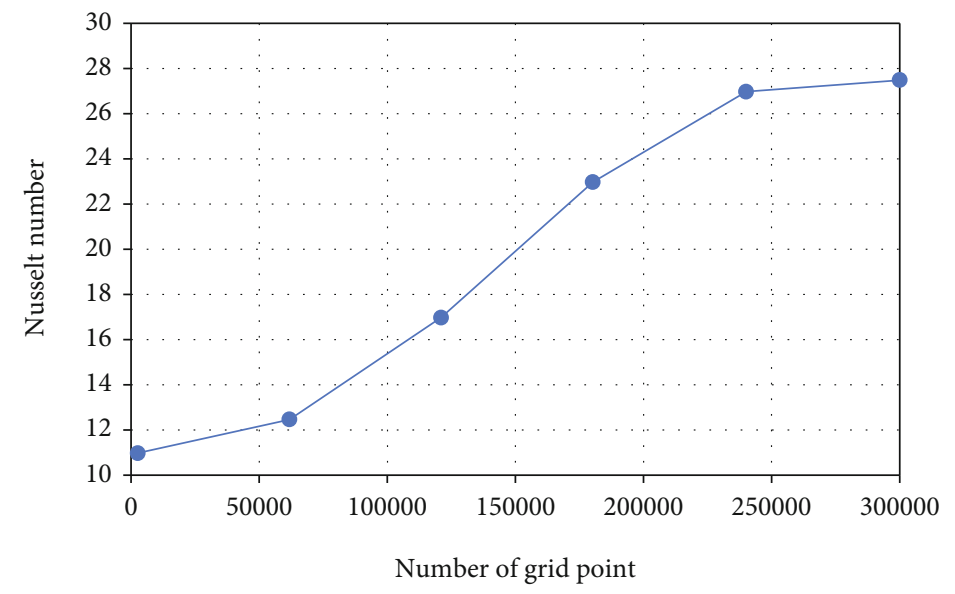

FIgURE 2: Changes in the Nusselt number with different numbers of networks.

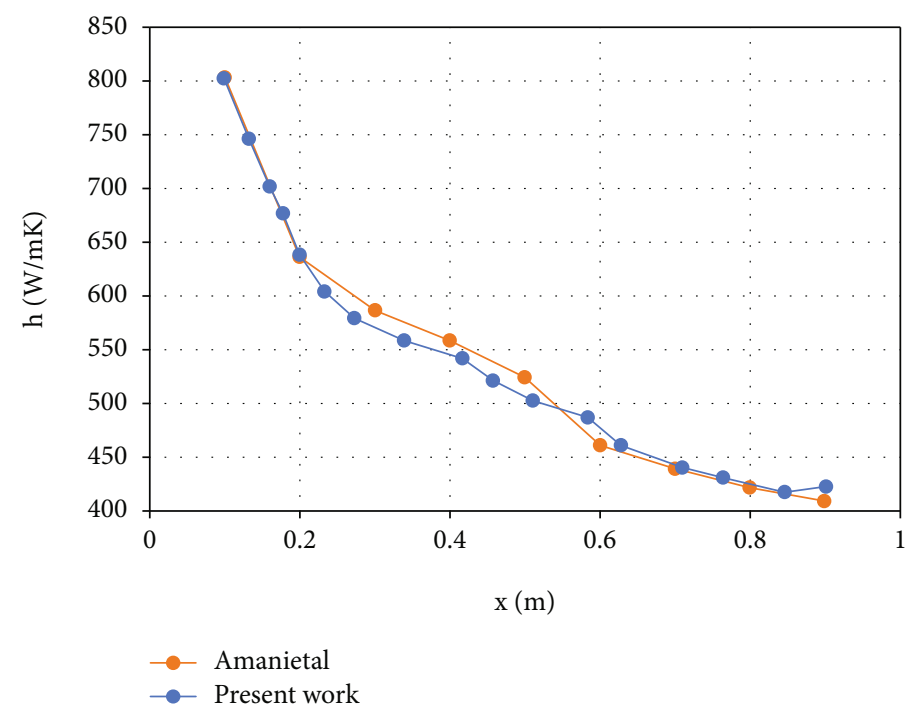

FIgURE 3: Check the validity of the results with Amani et al.

$q^{\prime \prime}$

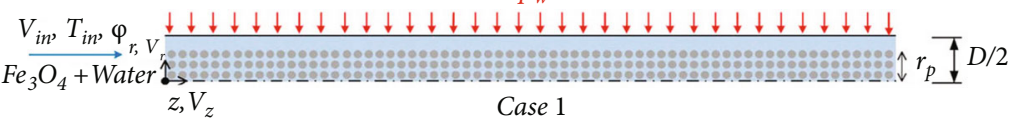

$\because$ Saturated porous medium

Clear ferrofluid

Figure 4: Geometrics description of the problem.

$$
\begin{gathered}
k_{\mathrm{hnf}}=\frac{k_{\mathrm{np}}+(n-1) k_{\mathrm{f}}-(n-1)\left(k_{\mathrm{f}}-k_{\mathrm{np}}\right) \phi}{k_{\mathrm{np}}+(n-1) k_{\mathrm{f}}+\left(k_{\mathrm{f}}-k_{\mathrm{np}}\right) \phi} k_{\mathrm{f}}, \\
\mu_{\mathrm{hnf}}=\frac{\mu_{\mathrm{f}}}{(1-\phi)^{2.5}} .
\end{gathered}
$$

2.3. Mesh Independence. Network generation is a crucial element of the simulation because of the influence of mesh on time, convergence, and solution outcomes. In addition, compared to an irregular network, a regular network has a better effect on the mentioned parameters. Significantly, networks close to the wall must be small enough to assess the slopes of the sloping physical properties in that area. A regular grid with incremental components throughout the radius was employed since these slopes are perpendicular to the walls. As illustrated in Figure 2, network independence is also demonstrated. In the network span, the comparative difference of Nusselt numbers was $1500 \times 50$ and $3000 \times$ 100 under 0.5 percent, as shown by Figure 2. In this case, 50 and 1500 networks were evaluated along the $Y$ and $X$ axes, respectively. As mentioned in the boundary condition problem, water enters the canal at $25^{\circ} \mathrm{C}$ and Reynolds 500 . 


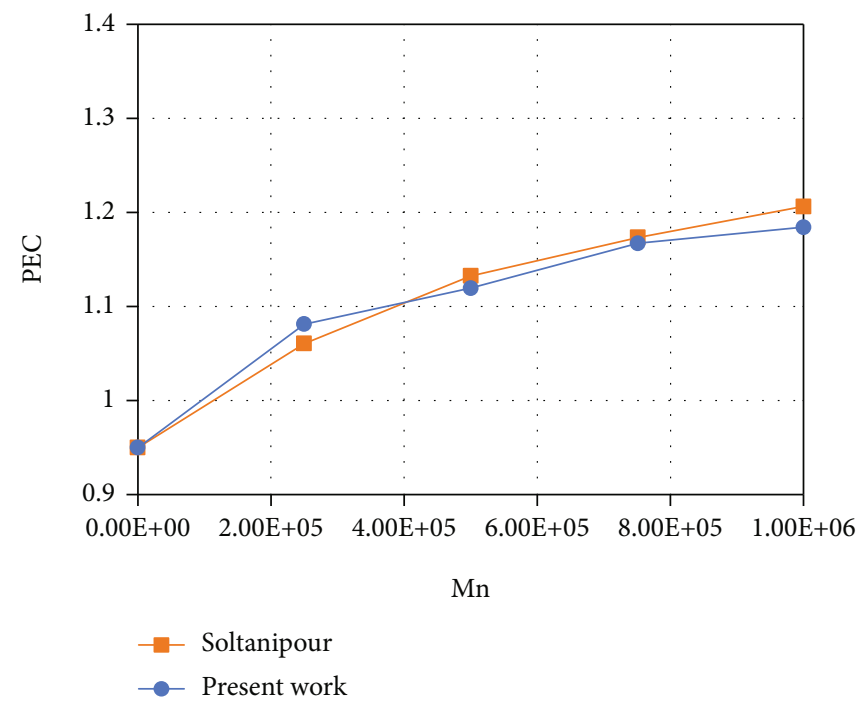

FIGURE 5: Check the validity of the results with Soltanipour and Pourfattah [23].

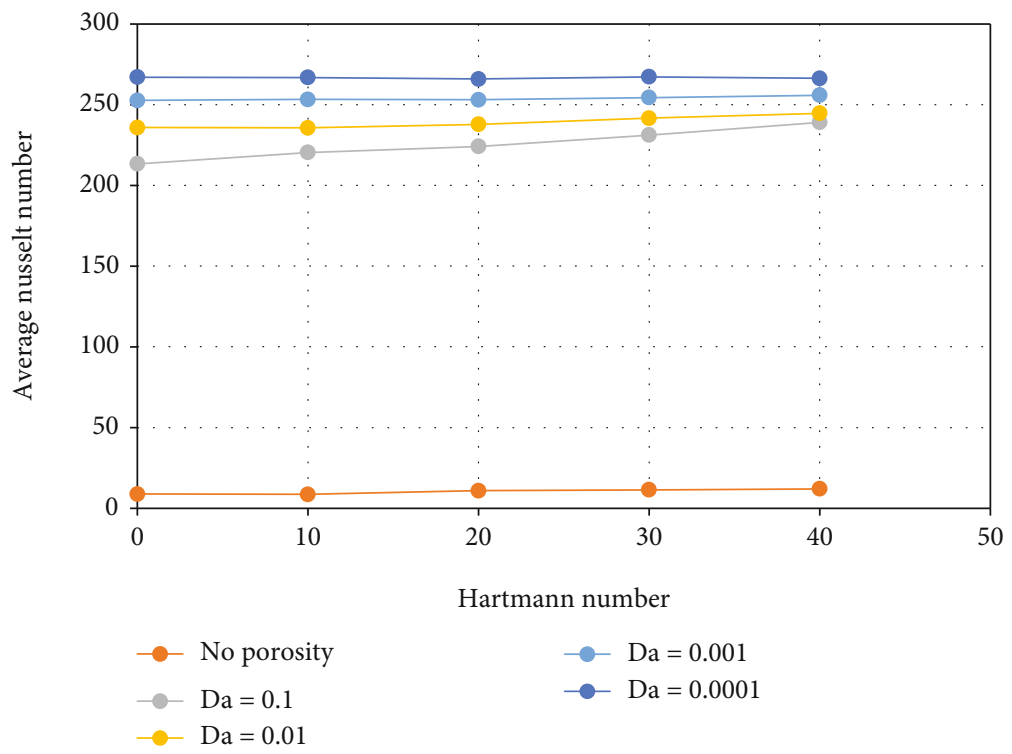

FIGURE 6: Average Nusselt number based on Hartmann number for different numbers of Darcy using water as the base fluid for 0.2 nanofluids.

2.4. Validation. Before reviewing the results, it is necessary to confirm the validity of the obtained results. In order to validate the numerical results, the heat transfer coefficient values provided by Amani et al. [23] in the porous metal foam tube under the constant magnetic field have been examined using the heat transfer convection subject $\mathrm{Fe}_{3} \mathrm{O}_{4}$ nanofluid. $\mathrm{Fe}_{3} \mathrm{O}_{4}$ nanofluid with a volume fraction of 0.2 and a diameter of 10 micrometers has been used. A nonuniform two-dimensional network is employed in this numerical research. Smaller networks are utilized in these regions because the temperature and velocity gradients between the pipe's intake and surface are considerable. The volume control approach is used to discretize the collection of equations. For diffusion and convective terms, the basic technique is employed to link velocity and pressure, whereas the second-order upstream method is utilized. The simulation is run on a $30 \times 4$ grid. For the equations of continuity, motion, and energy, convergence criteria are set so that residuals are smaller than 5-10. The validity of the findings is examined in Figure 3.

In order to make the validation more accurate, it is necessary to compare this research with Soltanipour and Pourfattah [24]. In this paper, a semiporous pipe has diameter $D$ and length $L$, and the value of $L / D$ is 15 . The $\mathrm{Fe}_{3} \mathrm{O}_{4} /$ water magnetic nanofluid enters the tube at a uniform velocity $V_{\text {in }}$, temperature $T_{\text {in }}$, and particle void fraction $\Phi_{\text {in }}$. The tube wall receives a constant $q_{w}^{\prime}$ flux. In investigated geometry, as shown in Figure 4 , a porous layer with radius rp is located in the core of the tube.

To check the validity of both velocity and temperature fields, it is necessary to validate the PEC criterion of Soltanipour 


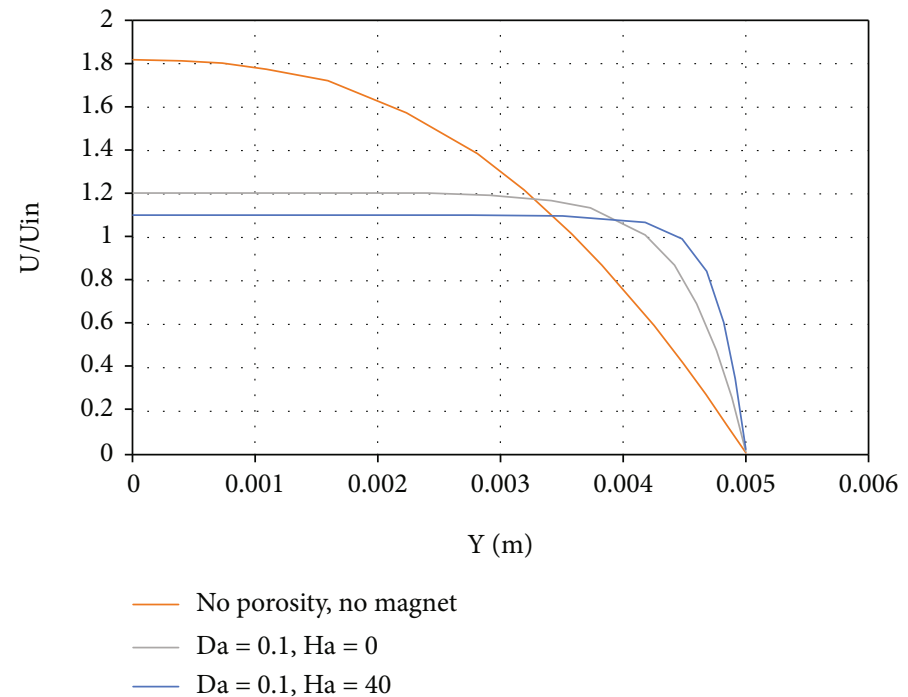

Figure 7: Dimensional dimensionless velocity versus radius of the cylinder for different inclination angles using water as base fluid.

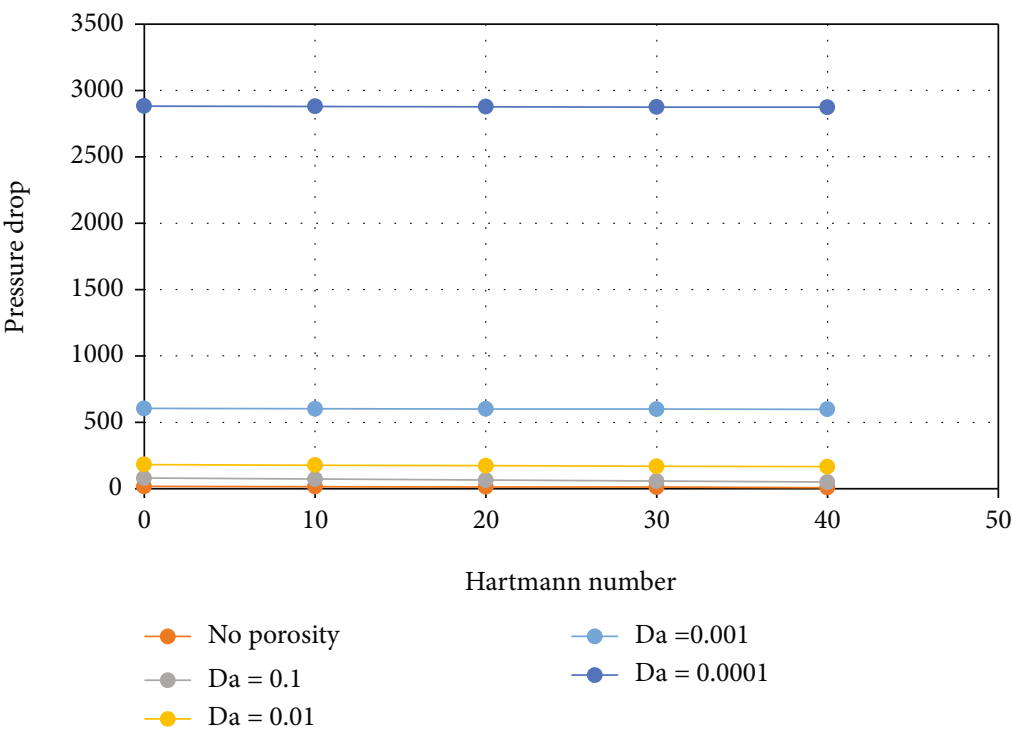

Figure 8: The impact of the Hartmann and Darcy numbers on the pressure drop in water and 0.2 nanofluids. The direction of the magnetic field is in the $y$-direction $(\psi=90)$.

and Pourfattah [24]. In Figure 5, the value of the PEC parameter in terms of the magnetic field is investigated. In this case, it is assumed that the porous medium occupies half the radius of the pipe.

According to the data obtained from comparing the results of Amani et al. [23] and Soltanipour and Pourfattah [24], the validity of the present study was confirmed.

\section{Results and Discussion}

3.1. Magnetic Field Effects on Heat Transfer. Heat transfer and flow behavior are both influenced by the magnetic field. By boosting the magnetic field, the flow can be stabilized and controlled. Figure 6 shows the difference in the mean of the Nusselt number vs. the Hartmann number in several bags with and without porosity. The direction of the magnetic field is in the $y$-direction $(\psi=90)$. The Hartmann number might be regarded as an improvement on the average Nusselt number, indicating an inhibitory effect of Lorentz force $(\vec{F}=\vec{J} \times \vec{B})$. The Hartmann number is the standard Lorentz volumetric force. An increment in it implies that the magnetic field is becoming more intense, furthermore increasing the Nusselt number by reducing the Darcy number from 0.1 to 0.0001 (as a consequence of diminishing the average permeability). In the absence of a porous medium, because nothing stops the flow of fluid and the velocity of the fluid 


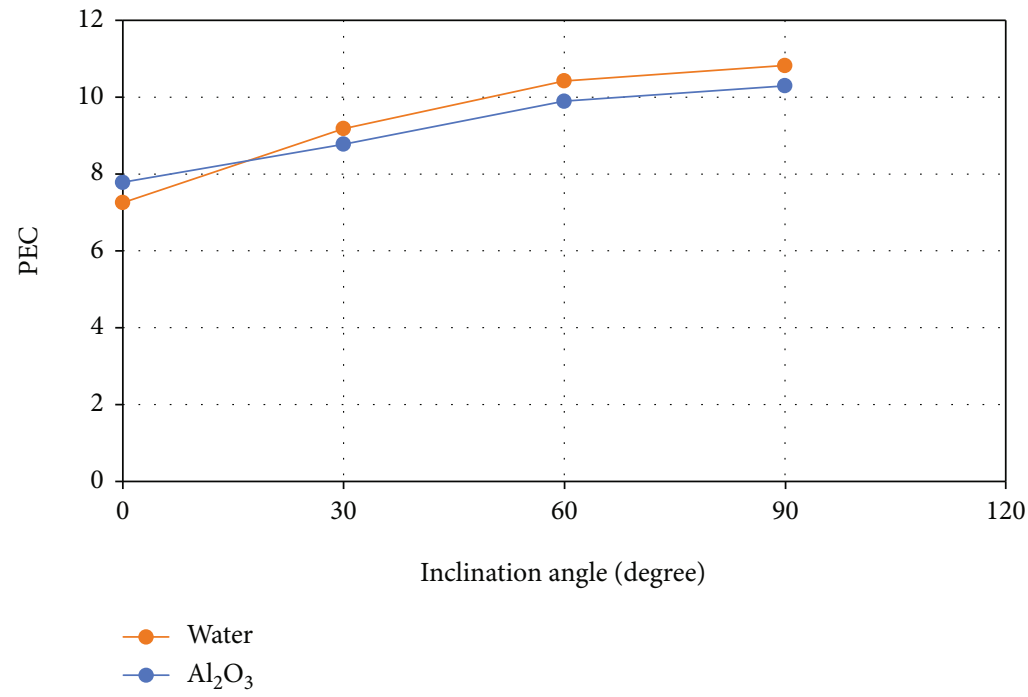

Figure 9: PEC for water and nanofluid 0.2 as a function of magnetic field direction $(\mathrm{Ha}=40, \mathrm{Da}=0.1)$.

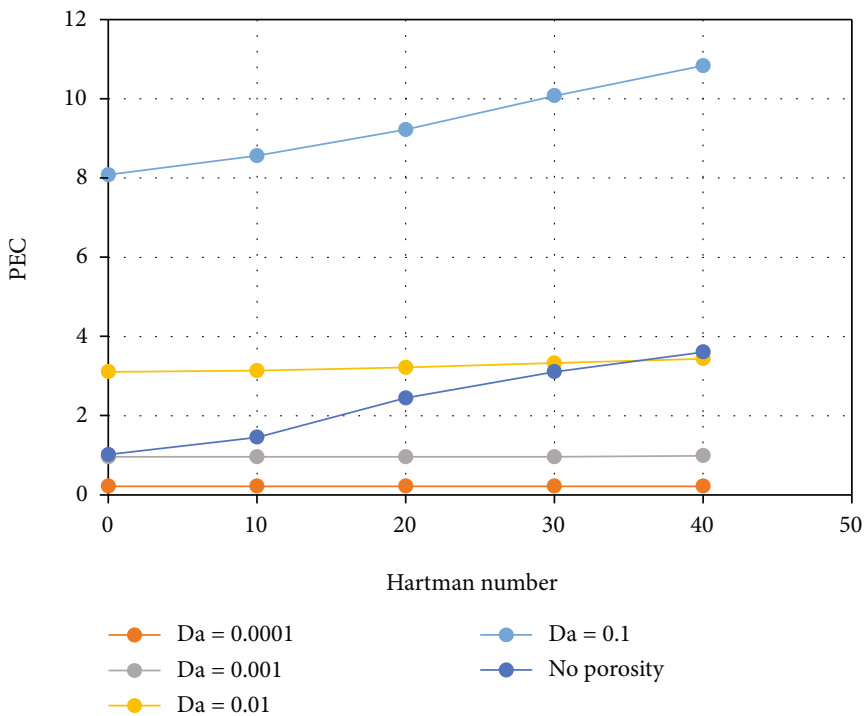

Figure 10: PEC changes against the effects of magnetic field and porosity using water as the base fluid for nanofluid 0.2 .

along the channel is not reduced, the amount of heat transfer is significantly reduced. As a result, heat transfer can be increased by simultaneously using the porous medium and the magnetic field.

The proportion of the input to the essential configuration speed is referred to as $U / U_{\text {in }}$ dimensionless relevant quantity. Figure 7 shows how the dimensional velocity ratio develops regarding the cylinder radius with water as the base fluid. Figure 7 additionally takes account of the influences of porosity and magnetic field.

It is noteworthy that in Figure 7, the ratio of speed without dimension decreases from 0 to 0.005 . Then, as the radius increases, it decreases further and finally reaches zero in the radius of 0.005 . Near the wall, high-speed slopes may also be seen (at $Y=0.005$ ). The average Nusselt number is increased by more significant velocity gradients in the wall, which increases the heat transfer rate. $U / U_{\text {in }}$ drops, as shown in Figure 7, using the magnetic field and porosity in the simulation at the beginning of the graph, the slope of the dimensionless velocity curve near the wall rises.

3.2. The Impacts of the Hartmann Number on Pressure Drop. Figure 8 depicts the influence of the Hartmann number on pressure drop, with pressure drops diminishing as the Hartmann number increases. This can be attributed to the heat transfer due to the magnetic field. This is due to the intensification of heat transfer due to the presence of a magnetic field. In addition, the pressure drop is shown by increasing the porosity from 0.0001 to 0.1 in the fixed Hartmann number. It has been observed that in the absence of a porous medium, because the medium does not block the fluid and 


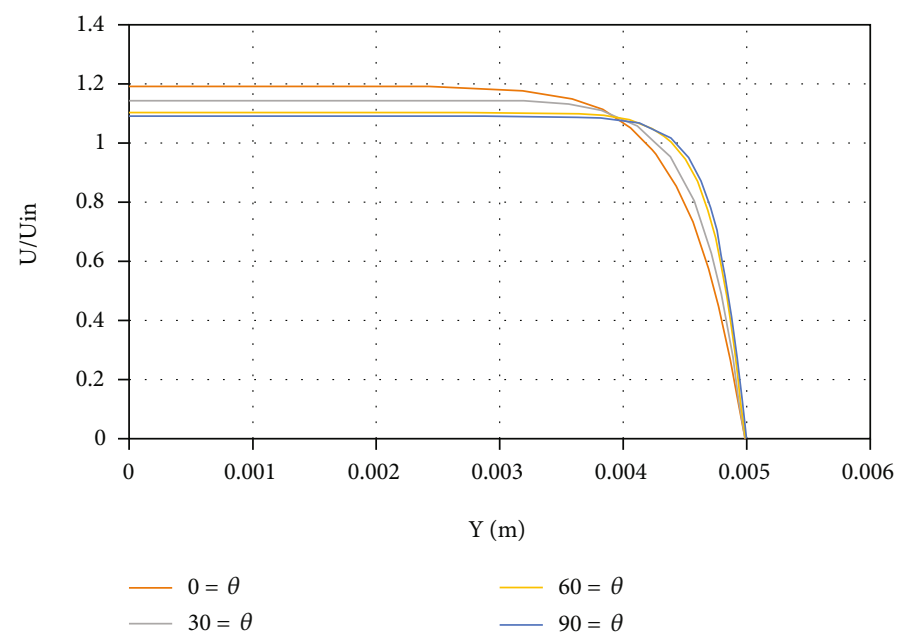

FIgURE 11: Dimensional dimensionless speed versus cylinder radius ratio for different magnetic field angles for $\mathrm{Al}_{2} \mathrm{O}_{3}$ nanofluid.

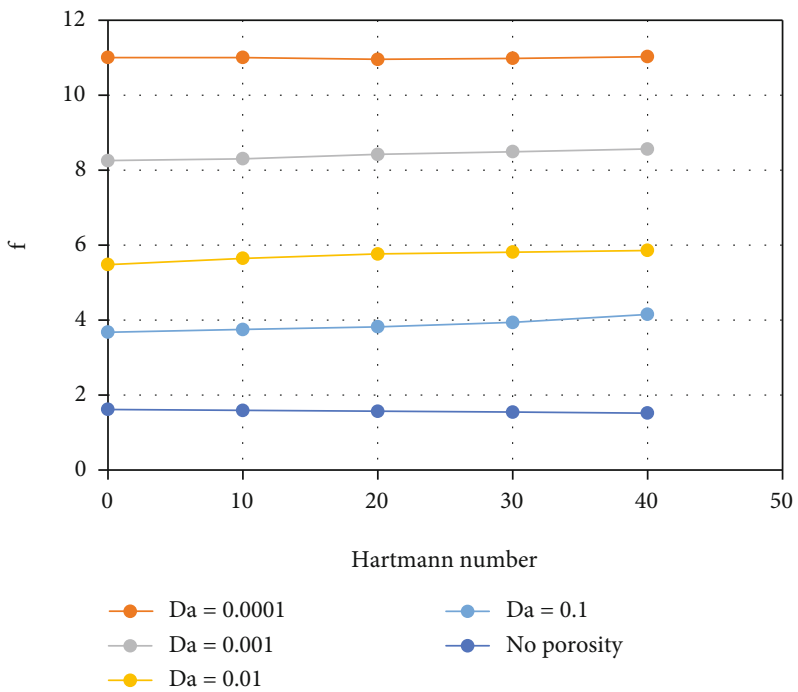

FIGURE 12: Effects of the Hartmann number on the friction factor at different Darcy numbers.

the velocity of the fluid is not decreasing, the amount of pressure is the lowest value compared to the other cases.

\subsection{Magnetic Field Orientation Impacts on Nanofluids. The} effect of the magnetic field orientation on the PEC (performance evaluation criterion) for a fluid comprising nanofluid is shown in Figure 9. The impact of mixing nanoparticles with the base fluid is also depicted. PEC increased somewhat with a shift in the orientation of the magnetic field from 0 to 90 degrees for all fluids, then dropped with an additional modification in orientation. This indicates a crucial orientation of the magnetic field. The magnetic field has the most significant impact on forced nanofluid convection in a chamber. According to the findings, the PEC in the investigated nanofluid was highest when the magnetic field was 90 degrees inclined. The proportion of the Nusselt number in each model of porosity and volume fraction of nanoparticles to the Nusselt number in the basic design, in the identical Reynolds number, is referred to as $\mathrm{Nu} / \mathrm{Nu}_{b}$. The function of the porosity approach or the composition of nanoparticles in improving heat transfer can be better understood using this ratio. Performance evaluation criteria (PEC) can be described as Equation (8) in this context:

$$
\mathrm{PEC}=\frac{\mathrm{Nu} / \mathrm{Nu}_{b}}{\left(\Delta P / \Delta P_{b}\right)^{1 / 3}}
$$

The PEC is applicable in comparing the hydrodynamic and thermal performance of the systems. Figure 10 presents the PEC values calculated for different Darcy numbers as a function of the Hartmann number. According to Figure 10, for a fixed Darcy number, an increment can be observed in the PEC due to Hartmann number enhancement which 


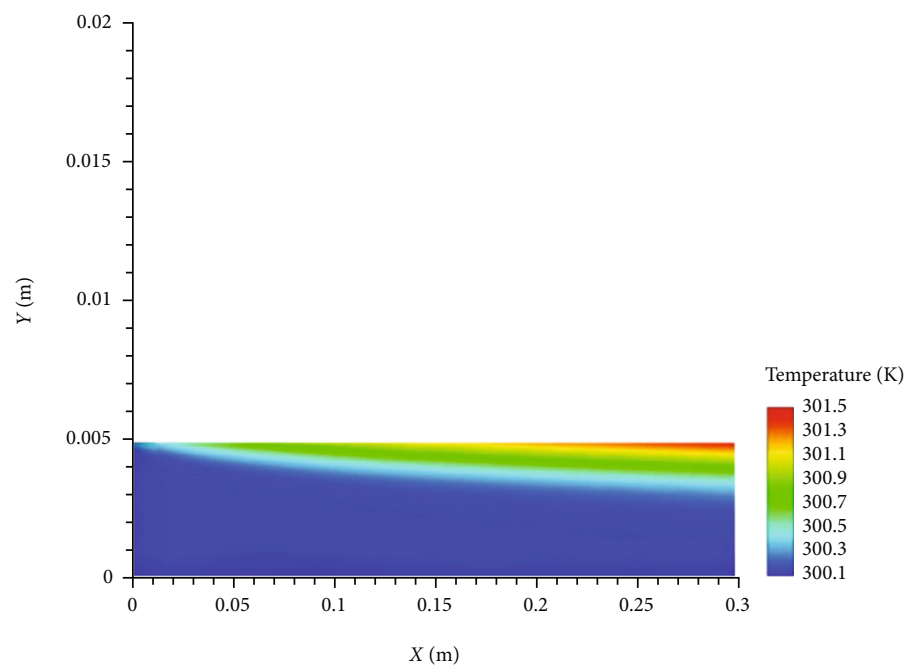

(a)

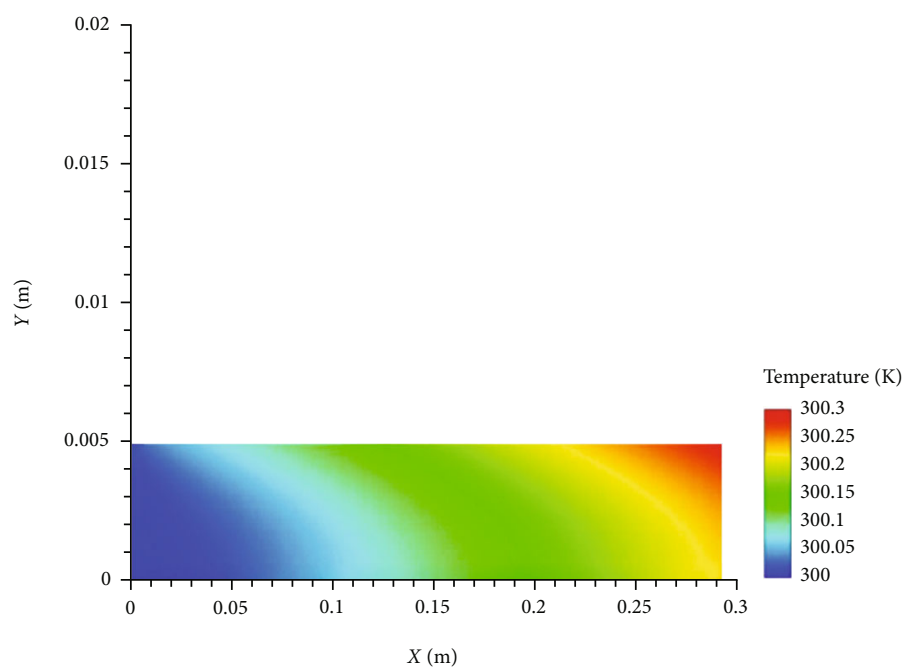

(b)

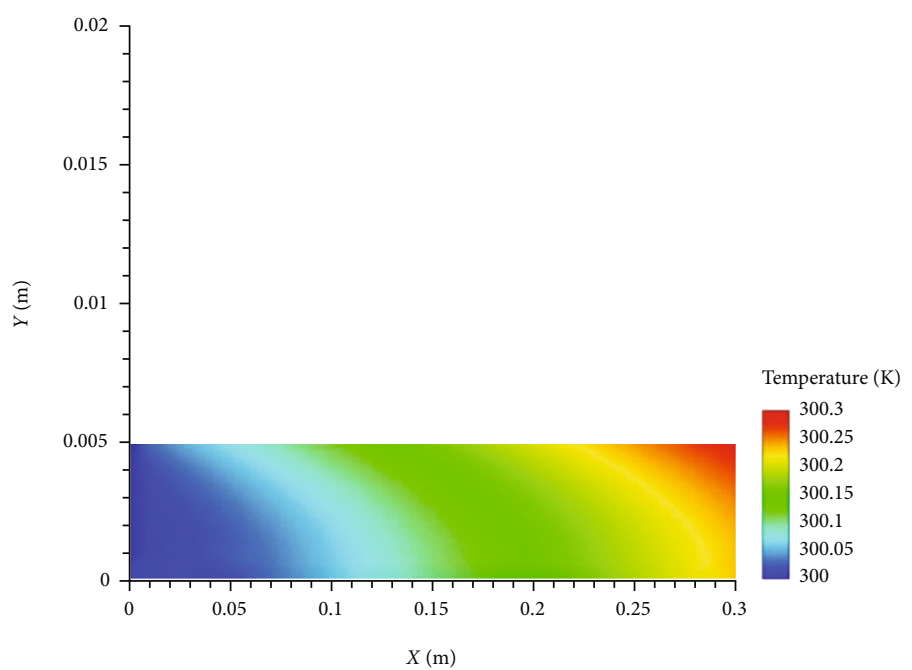

(c)

Figure 13: (a) Temperature contour for different Darcy and Hartmann numbers for water and nanofluid $0.2 \%(\mathrm{Da}=0, \mathrm{Ha}=0$ ). (b) Temperature contour for different Darcy and Hartmann numbers for water and nanofluid 0.2\% $(\mathrm{Da}=0.1, \mathrm{Ha}=0)$. (c) Temperature contour for different Darcy and Hartmann numbers for water and nanofluid $0.2 \%(\mathrm{Da}=0.1, \mathrm{Ha}=40)$. 


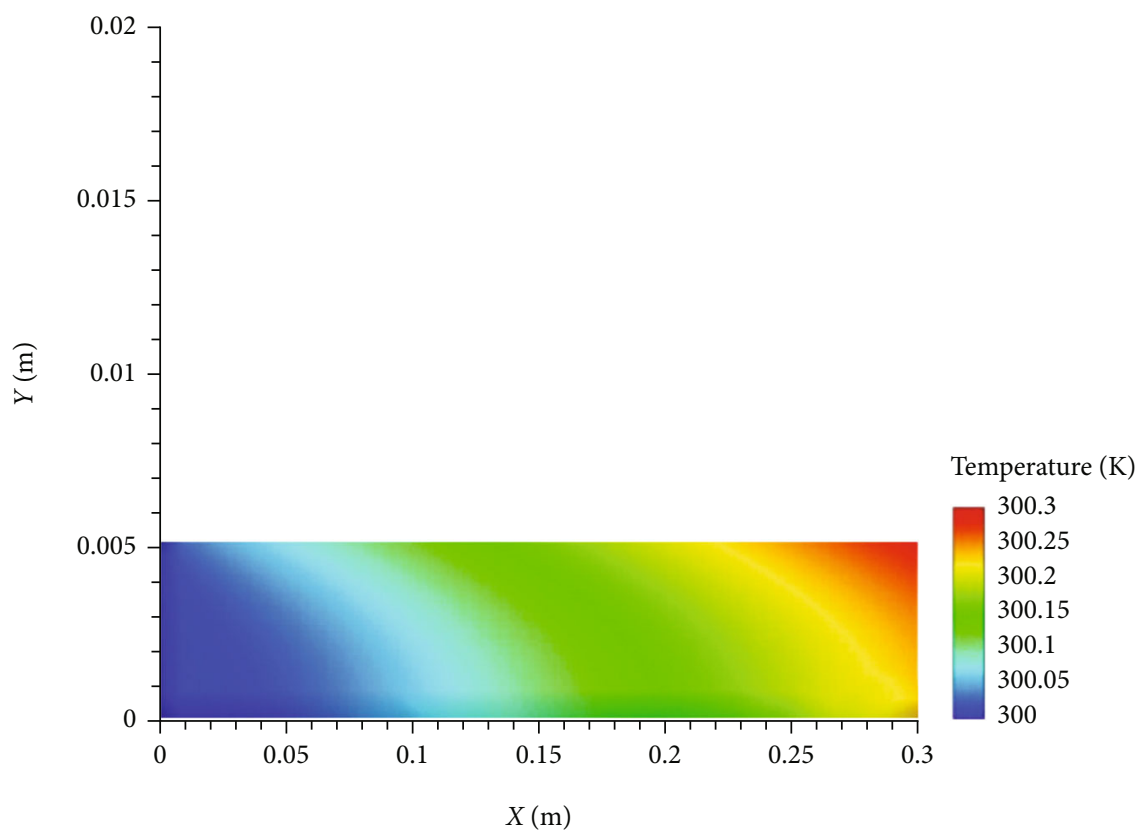

(a)

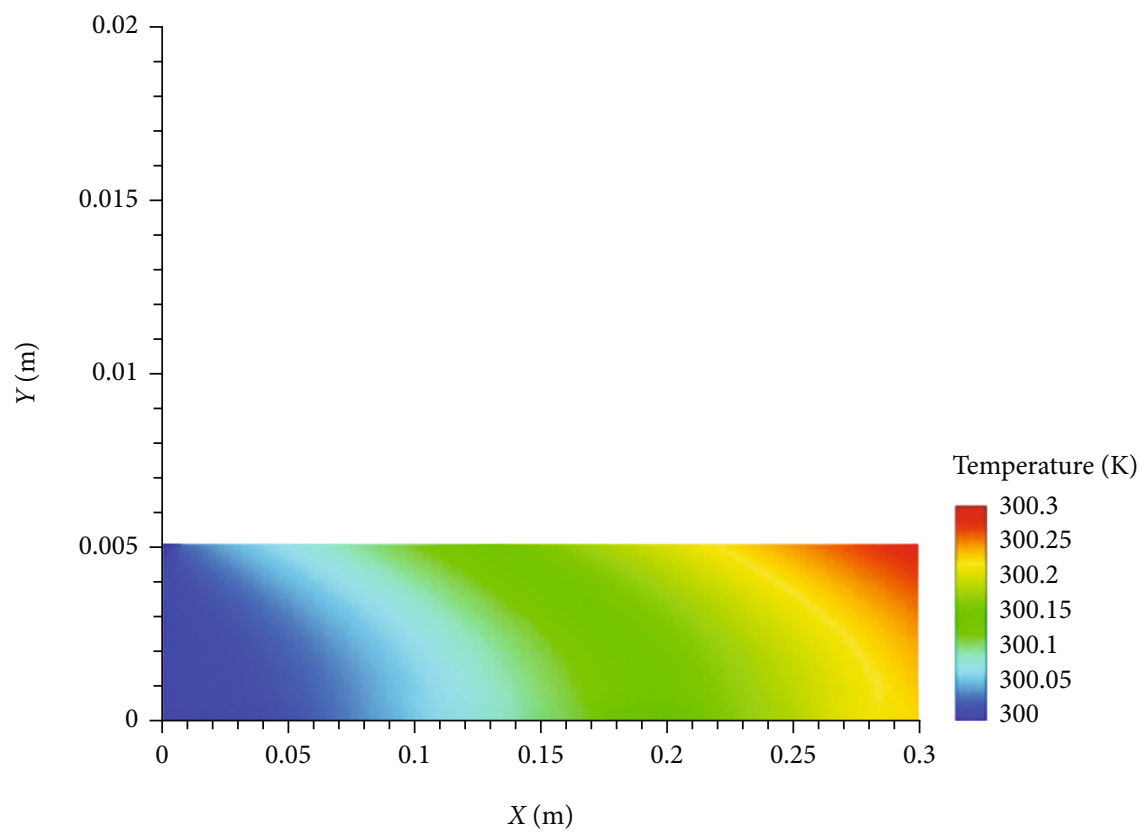

(b)

FIgURE 14: Continued. 


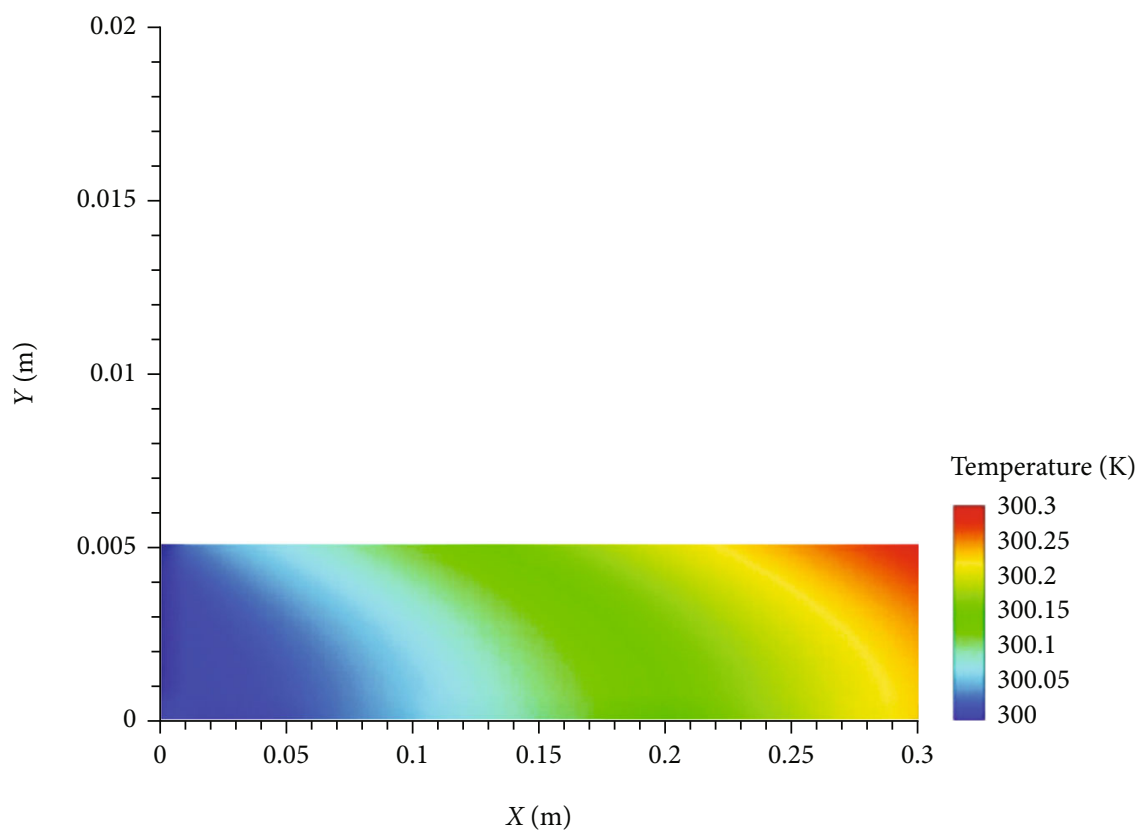

(c)

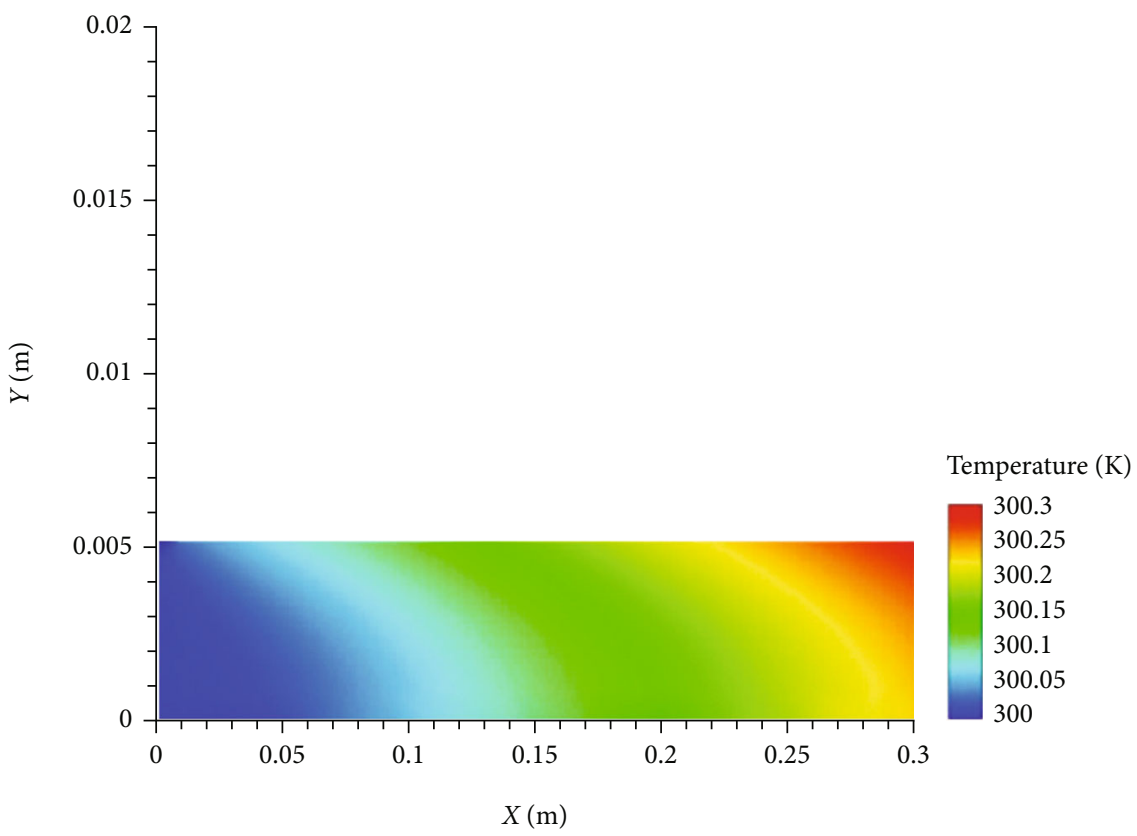

(d)

FIGURE 14: (a) Temperature contour for various slope angles for $\mathrm{Al}_{2} \mathrm{O}_{3}$ nanofluid $\left(\mathrm{Da}=0.1, \mathrm{Ha}=40, \psi=0^{\circ}\right)$. (b) Temperature contour for various slope angles for $\mathrm{Al}_{2} \mathrm{O}_{3}$ nanofluid $\left(\mathrm{Da}=0.1, \mathrm{Ha}=40, \psi=30^{\circ}\right)$. (c) Temperature contour for various slope angles for $\mathrm{Al}_{2} \mathrm{O}_{3}$ nanofluid $\left(\mathrm{Da}=0.1, \mathrm{Ha}=40, \psi=60^{\circ}\right)$. (d) Temperature contour for various slope angles for $\mathrm{Al}_{2} \mathrm{O}_{3}$ nanofluid $\left(\mathrm{Da}=0.1, \mathrm{Ha}=40, \psi=90^{\circ}\right)$.

could be assigned to the impact of Lorentz forces on the flow. Maximum PEC (10.81) was recorded at Darcy and Hartmann numbers of 0.1 and 40 , respectively. Thus, these values were taken as the default values for PEC calculations and investigation of the impact of magnetic field orientation for various nanofluid compositions.

Figure 11 depicts the dimensional velocity ratio variations for $\mathrm{Al}_{2} \mathrm{O}_{3}$ nanofluid as a function of cylinder radius. In Figure 11, the impact of the slope angle is also studied. The velocity ratio without dimension diminishes from 0 to 0.005 , then lowers again as the radius increases, as seen in Figure 9. Finally, when the radius becomes 0.005 , the relative velocity reaches zero. As depicted in Figure 11, increasing the inclination angle from $0^{\circ}$ to $90^{\circ}$ at the beginning of the curve decreases the dimensionless velocity ratio but increases close the $U / U_{\text {in }}$ slope wall. As shown in Figure 11, the magnetic field affects the Nusselt number in each direction. The magnetic field is more effective when applied vertically $\left(90^{\circ}\right)$ than in other directions. In addition, the higher the Nusselt number, the higher the PEC. Therefore, the PEC enhance. 
3.4. The Impact of Hartmann and Darcy Numbers in Friction Factor. Figure 12 compares the effect of the Hartmann number on the coefficient of friction. The increasing Hartmann number and decreasing Darcy number leads to increasing the coefficient of friction, which is attributed to the highvelocity slope. As a result, the shear stresses of the wall are intensified. For example, by placing a porous medium with $\mathrm{Da}=10^{-4}$, the coefficient of friction increases by about 7.2 times. However, the effect of the Hartmann number is negligible compared to the Darcy number. In other words, the application of the Hartmann number has no significant effect on current losses.

3.5. The Impact of Hartmann and Darcy Numbers in Temperature Counters. Figure 13(a) shows temperature lines for water as the base fluid in various Darcy and Hartmann values. According to Figure 13(b), the heat transfer rate is improved using the magnetic field and porosity.

In the lack of a magnetic field and a porous nickel media, temperature variations are small and thermal performance is minor, as illustrated in Figure 13(c). As the Darcy and Hartmann values rise, the percentage of convection in the overall heat transfer velocity rises. Furthermore, when Lorentz forces and the permeability of the environment grow, the convection term gets stronger. When a result, as Darcy and Hartmann's numbers rise, the temperature gradient near the wall rises. Better heat transfer performance is achieved with a thin thermal boundary layer close to the wall.

3.6. Impacts of Magnetic Field Direction on Temperature Contours. Figures 14(a)-14(d) depict the temperature profile of $\mathrm{Al}_{2} \mathrm{O}_{3}$ as the nanofluid at the maximum Darcy and Hartmann numbers, 0.1 and 40 , respectively.

As shown in Figures 14(a)-14(d), the temperature profile improves when the slope angle changes from $0^{\circ}$ to $90^{\circ}$. Also, a maximum temperature gradient is created for the $90^{\circ}$ mode. As previously stated, a thin thermal boundary layer near the wall allows for improved heat transfer.

\section{Conclusion}

The effects of mixing a magnetic field and a nanofluid $\left(\mathrm{Al}_{2} \mathrm{O}_{3}\right)$ into the water in a porous nickel medium on heat transfer were examined in this work. The use of cylindrical geometry, where the walls are subjected to constant and uniform heat flux, was investigated. A two-phase mixed model using a two-phase technique was employed to model the nanofluid flow. The following are some of the study's findings:

(i) The average Nusselt number rises with decreasing Darcy number and increasing Hartmann number

(ii) The pressure drop with increasing Hartmann and Darcy numbers shows a downward pattern

(iii) Improving PEC can be achieved by increasing the Hartmann and Darcy numbers (iv) In all cases investigated, adding additional metal nanofluids to the base fluid enhances the mean Nusselt number and PEC

(v) The PEC achieves its most excellent value for permeable porous medium (for example, media with $\mathrm{Da}=0.1$ and $\mathrm{Ha}=40$ )

(vi) Increasing the orientation of the magnetic field to a certain value (90 degrees) leads to an increase in PEC, and then, increasing the orientation angle reduces the PEC value. The magnetic field near $90^{\circ}$ leads to the highest PEC in the nanofluid under study

\section{Symbols}

$B: \quad$ The intensity of the magnetic field

C: $\quad$ Specific heat $\left(\mathrm{J} \mathrm{kg}^{-1} \mathrm{~K}^{-1}\right)$

g: $\quad$ Gravity acceleration $\left(\mathrm{m} \mathrm{s}^{-2}\right)$

J: $\quad$ Electric current density

K: $\quad$ Permeability of porous medium $\left(\mathrm{m}^{2}\right)$

$k$ : Thermal conductivity $\left(\mathrm{W} \mathrm{m}^{-1} \mathrm{~K}^{-1}\right)$

L: $\quad$ Length $(\mathrm{m})$

$P: \quad$ Dimensionless pressure

$p: \quad$ Pressure $(\mathrm{Pa})$

$q^{\prime \prime}$ : Heat flux $\left(\mathrm{W} \mathrm{m}^{-2}\right)$

$R: \quad$ Radius (m)

$T: \quad$ Temperature (K)

$U, V$ : Dimensionless velocity

$u, v: \quad$ Velocity components $\left(\mathrm{m} \mathrm{s}^{-1}\right)$

$X, Y$ : Dimensionless cylindrical coordinates

$x, y: \quad$ Cylindrical coordinates $(\mathrm{m})$.

Greek Symbols

$\alpha$ : Thermal diffusivity $\left(\mathrm{m}^{2} \mathrm{~s}^{-1}\right)$

$\beta$ : Thermal expansion coefficient $\left(\mathrm{K}^{-1}\right)$

$\varepsilon$ : Porosity

$\theta$ : Dimensionless temperature

9: Kinematic viscosity $\left(\mathrm{m}^{2} \mathrm{~s}^{-1}\right)$

$\mu$ : Dynamic viscosity $\left(\mathrm{kg} \mathrm{m}^{-1} \mathrm{~s}^{-1}\right)$

$\rho$ : Density $\left(\mathrm{kg} \mathrm{m}^{3}\right)$

$\sigma$ : Electrical conductivity $\left(\Omega^{-1} \mathrm{~m}^{-1}\right)$

$\varphi$ : Volume fraction

$\psi$ : Magnetic field angle.

\section{Abbreviations}

Da: Darcy number

Ha: Hartmann number

MHD: Magnetohydrodynamics

$\mathrm{Nu}$ : Nusselt number

Pr: $\quad$ Prandtl number

Re: Reynolds number.

\section{Data Availability}

The manuscript is a numerical and mathematical simulation of CFD equations. Therefore, there is not any dataset for the manuscript. 


\section{Conflicts of Interest}

We declare no conflict of interest.

\section{References}

[1] Q. Chen, J.-H. Hao, and T. Zhao, "An alternative energy flow model for analysis and optimization of heat transfer systems," International Journal of Heat and Mass Transfer, vol. 108, pp. 712-720, 2017.

[2] Z. Shah, M. R. Hajizadeh, N. A. Alreshidi, W. Deebani, and M. Shutaywi, "Entropy optimization and heat transfer modeling for Lorentz forces effect on solidification of NEPCM," International Communications in Heat and Mass Transfer, vol. 117, article 104715, 2020.

[3] P. H. Jadhav, G. Trilok, N. Gnanasekaran, and M. Mobedi, "Performance score based multi-objective optimization for thermal design of partially filled high porosity metal foam pipes under forced convection," International Journal of Heat and Mass Transfer, vol. 182, article 121911, 2022.

[4] Y. Menni, A. J. Chamkha, and A. Azzi, "Nanofluid transport in porous media: a review," Special Topics \& Reviews in Porous Media: An International Journal, vol. 10, no. 1, pp. 49-64, 2019.

[5] Y. Cui, J. Zhu, S. Zoras, and J. Zhang, "Comprehensive review of the recent advances in PV/T system with loop-pipe configuration and nanofluid," Renewable and Sustainable Energy Reviews, vol. 135, p. 110254, 2021.

[6] M. Sheikholeslami and H. B. Rokni, "Simulation of nanofluid heat transfer in presence of magnetic field: a review," International Journal of Heat and Mass Transfer, vol. 115, pp. 12031233, 2017.

[7] S. Yekani Motlagh, A. Sharifi, M. Ahmadi, and H. Badfar, "Presentation of new thermal conductivity expression for $\mathrm{Al}_{2} \mathrm{O}_{3}$-water and $\mathrm{CuO}$-water nanofluids using gene expression programming (GEP)," Journal of Thermal Analysis \& Calorimetry, vol. 135, no. 1, 2019.

[8] Y. He, Y. Jin, H. Chen, Y. Ding, D. Cang, and L. Huilin, "Heat transfer and flow behaviour of aqueous suspensions of $\mathrm{TiO}_{2}$ nanoparticles (nanofluids) flowing upward through a vertical pipe," International Journal of Heat and Mass Transfer, vol. 50, no. 11-12, pp. 2272-2281, 2007.

[9] M. U. Sajid and H. M. Ali, "Recent advances in application of nanofluids in heat transfer devices: a critical review," Renewable and Sustainable Energy Reviews, vol. 103, pp. 556-592, 2019.

[10] S. Nazari and D. Toghraie, "Numerical simulation of heat transfer and fluid flow of water-CuO nanofluid in a sinusoidal channel with a porous medium," Physica E: Low-dimensional Systems and Nanostructures, vol. 87, 2017.

[11] A. Sharifi, S. Y. Motlagh, and H. Badfar, "Investigation of the effects of two parallel wires' non-uniform magnetic field on heat and biomagnetic fluid flow in an aneurysm," International Journal of Computational Fluid Dynamics, vol. 32, no. 4-5, pp. 248-259, 2018.

[12] N. Dukhan and K.-C. Chen, "Heat transfer measurements in metal foam subjected to constant heat flux," Experimental Thermal and Fluid Science, vol. 32, no. 2, pp. 624-631, 2007.

[13] V. V. Calmidi and R. L. Mahajan, "Forced convection in high porosity metal foams," Journal of Heat Transfer, vol. 122, no. 3, pp. 557-565, 2000.
[14] B. Boyd and K. Hooman, "Air-cooled micro-porous heat exchangers for thermal management of fuel cells," International Communications in Heat and Mass Transfer, vol. 39, no. 3, pp. 363-367, 2012.

[15] M. Odabaee and K. Hooman, "Application of metal foams in air-cooled condensers for geothermal power plants: an optimization study," International Communications in Heat and Mass Transfer, vol. 38, no. 7, pp. 838-843, 2011.

[16] Y. R. Lin, J. H. Du, W. Wu, L. C. Chow, and W. Notardonato, "Experimental study on heat transfer and pressure drop of recuperative heat exchangers using carbon foam," Journal of Heat Transfer, vol. 132, no. 9, 2010.

[17] Y. Chen, P. Luo, Q. Tao, X. Liu, and D. He, "Natural convective heat transfer investigation of nanofluids affected by electrical field with periodically changed direction," International Communications in Heat and Mass Transfer, vol. 128, p. 105613, 2021.

[18] C. Qi, J. Tang, Z. Ding, Y. Yan, L. Guo, and Y. Ma, "Effects of rotation angle and metal foam on natural convection of nanofluids in a cavity under an adjustable magnetic field," International Communications in Heat and Mass Transfer, vol. 109, p. 104349, 2019.

[19] A. Izadi, M. Siavashi, H. Rasam, and Q. Xiong, "MHD enhanced nanofluid mediated heat transfer in porous metal for CPU cooling," Applied Thermal Engineering, vol. 168, p. 114843, 2020.

[20] P. Rabbani, A. Hamzehpour, M. Ashjaee, M. Najafi, and E. Houshfar, "Experimental investigation on heat transfer of $\mathrm{MgO}$ nanofluid in tubes partially filled with metal foam," Powder Technology, vol. 354, pp. 734-742, 2019.

[21] M. Ameri, M. Amani, and P. Amani, "Thermal performance of nanofluids in metal foam tube: thermal dispersion model incorporating heterogeneous distribution of nanoparticles," Advanced Powder Technology, vol. 28, no. 10, pp. 2747-2755, 2017.

[22] H. Badfar, S. Y. Motlagh, and A. Sharifi, "Numerical simulation of magnetic drug targeting to the stenosis vessel using $\mathrm{Fe}_{3} \mathrm{O}_{4}$ magnetic nanoparticles under the effect of magnetic field of wire," Cardiovascular Engineering and Technology, vol. 11, no. 2, pp. 162-175, 2020.

[23] M. Amani, M. Ameri, and A. Kasaeian, "Investigating the convection heat transfer of $\mathrm{Fe}_{3} \mathrm{O}_{4}$ nanofluid in a porous metal foam tube under constant magnetic field," Experimental Thermal and Fluid Science, vol. 82, pp. 439-449, 2017.

[24] H. Soltanipour and F. Pourfattah, "Simultaneous use of nonuniform magnetic field and porous medium for the intensification of convection heat transfer of a magnetic nanofluid inside a tube," Journal of the Brazilian Society of Mechanical Sciences and Engineering, vol. 43, no. 10, pp. 1-19, 2021. 